\title{
Estudo sobre os ambientes favoráveis à produção da líquida não-lateral /r/ no tratamento do desvio fonológico
}

\section{Study of the favorable environment to the production of the non-lateral liquid /r/ in the phonological treatment}

\author{
Márcia Keske-Soares ${ }^{1}$, Helena Bolli Mota ${ }^{2}$, Karina Carlesso Pagliarin ${ }^{3}$, Marizete Ilha Ceron ${ }^{4}$
}

\begin{abstract}
RESUMO
Os ambientes favoráveis são contextos facilitadores para a aquisição do segmento tratado. Eles podem ser analisados sob os seguintes aspectos: tonicidade, contexto antecedente e vogal seguinte. O objetivo deste relato de caso foi analisar a influência das variáveis lingüísticas (ambiente favorável) no tratamento de um sujeito com desvio fonológico evolutivo. Participou do estudo um sujeito com desvio fonológico, com idade de 6:8 no início do tratamento, do sexo masculino, tratado pelo Modelo ABAB-Retirada e Provas Múltiplas. Foram analisados os dados de fala pré e pós-tratamento e as Provas Alvo Básicas do primeiro ciclo de tratamento. Nas palavras-alvo selecionadas para terapia, foram analisadas as aquisições quanto ao alvo de tratamento, a líquida não-lateral /r/, e os ambientes favoráveis à aquisição no que se refere à tonicidade e aos contextos (antecedente e seguinte). Observou-se que o /r/, nas palavras-alvo selecionadas, não era encontrado em ambientes mais favoráveis; em geral, encontrava-se em ambiente neutro ou menos favorecedor. No entanto, verificou-se generalização no que se refere à aquisição da líquida não-lateral /r/ em Onset Medial, e a produção do fonema nas palavras-alvo selecionadas foi efetiva, destacando-se as produções das palavras [ba'rata], [ko'́ruza] e [mórãgu], que apresentaram maior número de ocorrências.
\end{abstract}

DESCRITORES: Fala; Distúrbios da fala/terapia; Fonoterapia; Criança; Transtornos da articulação

\section{INTRODUÇÃO}

O tratamento dos desvios fonológicos (DF) tem sido freqüentemente pesquisado em relação aos diferentes modelos terapêuticos e seus princípios teóricos. Estudos sobre a aquisição do fonema $/ \mathrm{r} / \mathrm{são}$ freqüentemente encontrados em crianças com aquisição normal e desviante, mas muitos não

(1) Doutora em Lingüística Aplicada pela Pontifícia Universidade Católica do Rio Grande do Sul - PUC-RS - Porto Alegre (RS), Brasil; Professora do Curso de Fonoaudiologia, da Especialização em Fonoaudiologia e do Curso de Mestrado em Distúrbios da Comunicação Humana da Universidade Federal de Santa Maria - UFSM - Santa Maria (RS), Brasil;

(2) Doutora em Lingüística Aplicada pela Pontifícia Universidade Católica do Rio Grande do Sul - PUC-RS - Porto Alegre (RS), Brasil; Professora do Curso de Fonoaudiologia, da Especialização em Fonoaudiologia e do Curso de Mestrado em Distúrbios da Comunicação Humana da Universidade Federal de Santa Maria - UFSM - Santa Maria (RS), Brasil;

(3) Acadêmica do Curso de Fonoaudiologia da Universidade Federal de Santa Maria - UFSM - Santa Maria (RS), Brasil; Bolsista PIBIC/CNPq.

(4) Acadêmica do Curso de Fonoaudiologia da Universidade Federal de Santa Maria - UFSM - Santa Maria (RS), Brasil; Bolsista PIBIC/CNPq.

Trabalho realizado na Universidade Federal de Santa Maria - UFSM - Santa Maria (RS), Brasil.

Endereço para correspondência: Márcia Keske-Soares. RST 287 (Faixa Nova de Camobi), 900, Cerrito, Santa Maria - RS, CEP 97060-500. E-mail: keske-soares@uol.com.br

Recebido em: 20/12/2005; Aceito em: 19/12/2006 consideram o ambiente favorável para escolha das palavrasalvo para o tratamento.

Não se tem conhecimento de pesquisas correlacionando a líquida não-lateral /r/ e os ambientes facilitadores no tratamento de crianças com DF. Assim, a seleção das palavrasalvo é importante no que se refere à escolha das palavras de estímulo. Deve-se observar o contexto fonético, pois alguns contextos facilitam a produção correta dos sons-alvo e outros tornam a produção dificultosa. Quando se considera o contexto fonético, deve-se atentar para o acento, a posição na sílaba e na palavra, os sons adjacentes, o número de sons problemas no contexto, a presença de substituição de som similar foneticamente, além do número de outros sons e sílabas no contexto. A seleção das palavras-alvo, portanto, deve ser bastante criteriosa. Por isso, há necessidade de se estudar o ambiente favorável (AF) em relação às crianças com aquisição desviante, para que o resultado do tratamento se torne mais eficaz.

O objetivo deste relato de caso foi analisar a influência das variáveis lingüísticas (ambiente favorável) no tratamento de um sujeito com desvio fonológico evolutivo.

Uma das formas de estudar a linguagem oral é pesquisando a aquisição fonológica da criança. $\mathrm{O}$ aprendizado das palavras se inicia nos primeiros anos de vida e, entre quatro e cinco anos, a criança tem que ter todos os sons e seqüênci- 
as de sons de sua língua. A ordem de aquisição normal das consoantes do Português Brasileiro, quanto ao modo de articulação, são as nasais e plosivas, seguidas das fricativas e, finalmente, das líquidas, sendo que as líquidas laterais são adquiridas antes das não-laterais ${ }^{(1)}$.

Os "erres" são os sons que aparecem mais tarde no sistema fonológico da criança, por serem os mais complexos na aquisição. Um estudo da aquisição normal do 'r' e seu status fonológico ${ }^{(2)}$, realizado com 110 crianças falantes do Português com desenvolvimento fonológico normal e idades entre 2:0 a 3:9, demonstrou que o ' $r$ - fraco' deve ser adquirido entre 3:8 e 3:9. Quanto à posição na palavra, a posição de Coda Final (CF) é a primeira a ser adquirida por ser uma posição mais saliente, sendo seguida de Onset Simples (OS), Onset Medial (OM) e, por último, de Coda Medial (CM). A aquisição do 'r-forte' é anterior à do 'r-fraco', sendo esta concluída entre $2: 6$ a 2:7.

$\mathrm{O}$ desvio fonológico (DF) caracteriza-se pela desorganização, inadaptação ou anormalidade no sistema de sons da criança em relação ao sistema padrão de sua comunidade lingüística, inexistindo quaisquer comprometimentos orgâ$\operatorname{nicos}^{(3)}$. O DF, também denominado transtorno fonológico ${ }^{(4)}$, é definido como uma dificuldade de fala, caracterizada pelo uso inadequado de sons, de acordo com a idade e com variações regionais, que podem envolver erros na produção, percepção ou organização dos sons.

Nas desordens do aprendizado de alguns sons da fala, ou seja, nos DF, observa-se uma freqüência maior de alterações nas líquidas, principalmente das líquidas não-laterais /r/ e /R/. Em geral, essas alterações no sistema fonológico são caracterizadas por apagamento e/ou substituições dos sons ${ }^{(1)}$.

A aquisição desviante das líquidas não-laterais, estudadas ${ }^{(5)}$ em 78 crianças com DF, com idade entre 3:0 a 13:0, indica que as mesmas apresentam índices maiores de não produção em relação ao 'r-fraco'. Quanto à aquisição deste segmento, a posição na sílaba é semelhante, tanto para crianças com aquisição normal ${ }^{(2)}$ quanto para as com DF, verificando-se a seguinte ordem: OS, Coda e Onset Complexo (encontro consonantal). Em relação ao tipo de sílaba, as substituições são mais comuns do que as omissões em OS, e as omissões são mais freqüentes do que as substituições em Coda e em Onset Complexo.

A terapia fonológica deve enfatizar a adequação dos padrões fonológicos na fala da criança, sendo o principal de seus objetivos a generalização. Um dos modelos de terapia estudados com base na fonologia é o ABAB-Retirada e Provas Múltiplas, que tem como princípio a hierarquia implicacional, ou seja, considera que o ensino de um traço marcado implica a aquisição de traços menos marcados, sem tratamento direto. Esse modelo consta de ciclos de tratamento, com Provas Alvo Básicas (PABs), que mensuram a aquisição dos sons-alvo nas palavras-alvo. Ao final do ciclo, temse o Período de Retirada, que são sessões sem tratamento direto sob o som alvo. Neste período, são aplicadas Provas de Generalização (PG) para avaliar se os traços trabalhados nos sons-alvo selecionados, foram generalizados aos sons não treinados ${ }^{(3)}$.

A aplicabilidade da terapia com base fonológica utili- zando o Modelo ABAB-Retirada e Provas Múltiplas e o princípio da hierarquia pelo Modelo Implicacional de Complexidade de Traços (MICT) ${ }^{(6)}$, foram confirmados pelo estu$\mathrm{do}^{(3)}$ de 35 sujeitos com DF, com média de idade de 5:5. Os sujeitos tratados expandiram seu inventário fonético e sistema fonológico, melhorando a contrastividade e, conseqüentemente, a inteligibilidade da fala após o tratamento.

$\mathrm{O} \mathrm{MICT}^{(6)}$ apresenta as relações existentes entre os traços marcados na aquisição da complexidade segmental pelas crianças com DF, falantes do Português. O MICT é representado sob a forma de uma árvore, na qual a raiz representa um estado zero de complexidade, de onde partem caminhos para a aquisição dos traços marcados. Esse modelo constitui-se de três rotas (A, B e C) e são hierarquizados em nove níveis, que vão do estado zero até o nível nove, crescente em complexidade de traços.

A generalização estrutural obtida a partir do tratamento utilizando os róticos foi estudada ${ }^{(7)}$ em quatro sujeitos com DF, com idades entre 4:0 e 6:4, com comparação entre dois diferentes modelos de terapia (Modelo de Oposições Máximas Modificado e ABAB-Retirada e Provas Múltiplas), a fim de verificar a ocorrência dos diversos tipos de generalização. Esse estudo constatou que a generalização a itens não utilizados no tratamento e para outra posição na palavra ocorreu em dois sujeitos tratados com a líquida não-lateral /r/. Quanto à generalização dentro de uma classe de sons, obteve-se o resultado por três dos sujeitos pesquisados, sendo que o único sujeito a não apresentar esse tipo de generalização foi aquele tratado pelo Modelo de Oposições Máximas Modificado.

Um das maneiras de tornar a aquisição dos sons mais rápida é considerar o contexto fonológico facilitador, ou seja, $\mathrm{o}$ ambiente facilitador (AF). As pesquisas em relação aos AF para a aquisição do " $r$ " foram realizadas em crianças com aquisição fonológica normal. Considerando a líquida nãolateral /r/ em posição de Onset, tem-se os seguintes AF: tonicidade da sílaba e contexto facilitador, vogal antecedente e vogal seguinte ${ }^{(2,8-9)}$.

Um estudo ${ }^{(8)}$ referente ao AF para a produção do $/ \mathrm{r} / \mathrm{em}$ OS, indica que a sílaba tônica é apontada como mais facilitadora; entretanto, a sílaba postônica é referida como a menos facilitadora. Quanto ao contexto, a vogal /i/ que antecede o /r/ é apontada como a mais favorecedora para a produção correta deste seguimento, enquanto que esta também é a vogal favorecedora sucedendo o $/ \mathrm{r} /$.

Nesse aspecto, o estudo ${ }^{(9)}$ do processo de aquisição das consoantes líquidas em OS, realizado com 310 crianças com desenvolvimento fonológico típico, e idades entre 2:0 e 7:1, mostrou que a variável posição na estrutura das palavras foi significativa para a aquisição das líquidas do Português. Em relação ao contexto fonológico seguinte, o emprego da líquida não-lateral /r/ não se mostrou favorável por nenhuma das sete vogais. Quanto ao contexto fonológico precedente, a vibrante /r/ não foi favorecida pelas vogais. E quanto à variável tonicidade, a aquisição do /r/ mostra-se favorecida pela sílaba postônica.

$\mathrm{O}$ estudo $^{(10)}$ das variáveis lingüísticas que atuam na aquisição fonológica da líquida não-lateral /r/, indicando o nível 
de facilitação das palavras com este segmento, foram indicados no potencial de favorecimento dos vocábulos e sugeridas palavras para a utilização como palavras-estímulo lingüisticamente mais eficientes para terapia. Em outra pesquisa $^{(11)}$, o fonema $/ \mathrm{r}$ / foi indicado como mais favorecedor no ambiente com a vogal /i/ antecedente, sendo que o contexto seguinte e a tonicidade não foram favoráveis para a aquisição. Como fatores menos favorecedores estão, no ambiente antecedente a vogal / $/$ /, no ambiente seguinte as vogais /e/ e / / /, e na tonicidade, a sílaba postônica. Os demais ambientes foram considerados neutros, ou seja, no ambiente antecedente, as vogais /u/, /o/, /e, a, O/, e no ambiente seguinte as vogais /o/, /u/, /i, a/. Quanto à tonicidade, foram encontrados como fator neutro as sílabas tônica e pretônica.

Em um estudo ${ }^{(10)}$ realizado com a finalidade de especificar o nível de facilitação do surgimento do /r/ nas palavras, foram indicadas palavras lingüisticamente mais eficientes na reabilitação fonológica dos distúrbios da fala. Os padrões de facilitação do /r/ em OS foram divididos em 12 níveis hierárquicos, que foram indicados, considerando a posição que o segmento ocupa na sílaba e na palavra, o ambiente fonético antecedente e seguinte, a tonicidade da sílaba em que o segmento está inserido e o número de sílabas das palavras. Nestes foram indicadas palavras para cada padrão, quando houvesse no Português, sendo que, no padrão 1, as mais favorecedoras eram, por exemplo [pi'ru] e [pi'ruka], por apresentar-se em OS, estarem antecedidas pela vogal /i/ e em sílaba tônica.

As de menor favorecimento, indicadas pelo padrão que existam palavras estão no padrão 10 (['zeru], ['perula]), essas apresentam-se em OS, antecedidas pela vogal / $/ \varepsilon /$ e encontra-se em sílaba tônica. Quanto ao padrão 12, no qual não foram encontradas palavras na língua portuguesa, devem ser consideradas para a seleção das mesmas a harmonia vocálica, a palatalização e a redução de ditongo.

\section{APRESENTAÇÃO DO CASO CLÍNICO}

Este relato de caso constituiu-se de um sujeito com DF, com 6:8 de idade, do sexo masculino. O sujeito faz parte do banco de dados do Centro de Estudos de Linguagem e Fala (CELF) do projeto "Estudo comparativo da generalização em três modelos de terapia fonológica, em crianças com diferentes graus de severidade do desvio fonológico", registrado no Gabinete de Projetos (GAP) sob n 12650 , e no Comitê de Ética em Pesquisa (CEP) sob no 046/02.

O sujeito foi atendido no Serviço de Atendimento Fonoaudiológico (SAF) da Universidade Federal de Santa Maria - RS (UFSM), havendo autorização prévia dos pais/ responsáveis por intermédio da assinatura do termo de consentimento livre e esclarecido.

Primeiramente, o sujeito foi avaliado por meio de triagem fonoaudiológica, realizada no próprio serviço (SAF), em que foi diagnosticado o DF, sendo então encaminhado para o CELF, onde foi submetido a novas avaliações. O diagnóstico de DF foi confirmado por meio de avaliações fonoaudiológicas (Avaliação da Linguagem, Exame Articulatório, Avaliação do Sistema Estomatognático, Ava- liação da Discriminação Auditiva, Avaliação Psicomotora, Avaliação da Memória de Trabalho e Avaliação do Vocabulário) e complementares (Avaliação Otorrinolaringológica, Audiológica e Neurológica). Para a obtenção dos dados da fala utilizou-se a Avaliação Fonológica da Criança (AFC) $)^{(12)}$, e para análise do mesmo, utilizou-se a análise contrastiva e por traços distintivos.

Para a escolha do sujeito foi utilizado como critério fundamental que o mesmo não apresentasse alterações significativas nas avaliações realizadas, à exceção da avaliação fonológica. Esta revelou desordens no nível fonológico e inventário fonético reduzido, com comprometimento da inteligibilidade da fala.

A avaliação da linguagem foi realizada de modo informal, por meio de conversa espontânea a partir da execução de ordens simples e complexas, adequação das respostas, execução de ordens solicitadas, organização lógica do pensamento, estrutura gramatical das sentenças e vocabulário empregado.

No exame articulatório, foi observada a capacidade da criança em repetir as palavras conforme o modelo dado pelo terapeuta; os resultados encontrados confirmaram as alterações do AFC.

$\mathrm{Na}$ avaliação do sistema estomatognático, foram avaliados aspectos relacionados às estruturas e funções do mesmo, tendo sido realizada a fim de excluir a existência de quaisquer fatores orgânicos que pudessem interferir na produção dos sons da fala.

A discriminação auditiva foi avaliada com base no Teste de Figuras para Discriminação Auditiva, adaptado do The Boston University Speech Sound - Picture Discrimination Test (1990) $)^{(13)}$, a fim de avaliar a discriminação dos sons quanto ao ponto, modo de articulação e sonoridade.

$\mathrm{Na}$ avaliação psicomotora, foram observados os padrões do desenvolvimento psicomotor conforme a idade cronológica da criança, sendo avaliadas a coordenação visomotora, a coordenação dinâmica geral, o controle postural (equilíbrio) e a organização perceptiva.

$\mathrm{Na}$ avaliação da memória de trabalho, foi avaliada a capacidade da criança para lembrar e evocar palavras e dígitos emitidos pela terapeuta após curto intervalo de tempo.

Para a avaliação do vocabulário foi utilizado o teste ABFW - Teste de Linguagem Infantil nas Áreas de Fonologia, Vocabulário, Fluência e Pragmática, que tem como objetivo verificar a competência lexical da criança.

$\mathrm{Na}$ AFC, foram analisados os dados de fala, realizandose a análise contrastiva a qual se fundamenta na comparação entre o sistema fonológico da criança e o sistema padrão adulto. Delimitou-se o inventário fonético da criança, ou seja, sua capacidade articulatória. Os resultados da análise contrastiva permitiram determinar se os fonemas estavam ou não estabelecidos no sistema fonológico da criança. Para tanto, foram considerados os seguintes critérios $^{(14)}: 80 \%$ ou mais - seguimento estabelecido; $40 \%$ - 79\% - seguimento parcialmente estabelecido; $0 \%$ - 39\% - seguimento não estabelecido.

Após a avaliação realizada, a criança foi submetida ao tratamento pelo Modelo ABAB-Retirada e Provas Múlti- 
plas ${ }^{(15)}$, sendo selecionado como alvo, no primeiro ciclo, a líquida não-lateral /r/ em OM.

O tratamento pelo Modelo ABAB - Retirada e Provas Múltiplas iniciou-se pela coleta dos dados de fala (A1), realizada mediante gravação da nomeação e da fala espontânea, utilizando o AFC, seguido da transcrição e análise fonológica. Depois, foram determinados os traços distintivos alterados e, a partir disso, delimitado o som-alvo para o tratamento, bem como as respectivas palavras-alvo e as do bombardeio auditivo.

A intervenção terapêutica, no primeiro ciclo de tratamento (B1), teve duração de nove sessões, sendo realizadas duas sessões semanais de terapia fonoaudiológica, com duração de 45 minutos cada. Durante o ciclo, eram realizadas três Provas Alvo Básicas (PAB), na primeira, quinta e nona sessão, as quais avaliavam a aquisição do som-alvo nas palavras alvo e nas não-alvo. Ao término do tratamento (B1), foram realizadas cinco sessões do Período de Retirada (A2), que consiste de um intervalo sem tratamento direto sobre o som-alvo. Nestas sessões, eram realizadas coletas da nomeação e de fala espontânea, por meio do AFC, a fim de observar as generalizações obtidas quanto à aquisição de sonsalvo e não-alvo no sistema fonológico da criança.

Está esquematizado, no Quadro 1, o Modelo ABAB Retirada e Provas Múltiplas, considerando-se as provas nele contidas, para cada ciclo de tratamento as PAB e, no Período de Retirada, as PGs.

No caso em estudo, primeiramente, foi feita a análise do sistema fonológico, verificando-se os AF para a produção da líquida não-lateral /r/, considerando-se os resultados obtidos na coleta dos dados de fala (A1), denominada Avaliação Fonológica Inicial (AFI), em comparação aos resultados obtidos nas Prova de Generalização do Período de Retirada (A2), denominada Avaliação Fonológica Final (AFF).

As palavras-alvo selecionadas para terapia foram analisadas conforme os pressupostos teóricos quanto aos ambientes favoráveis mais propícios para a aquisição do som-alvo, considerando-se tonicidade, contexto seguinte e antecedente. Durante o tratamento, foram analisadas as produções corretas e incorretas, considerando-se o som-alvo /r/ nas palavras-alvo selecionadas para as PABs.

O Quadro 2 descreve a avaliação fonológica inicial (prétratamento) e final (pós-tratamento) do sujeito deste relato de caso.

$\mathrm{Na}$ AFI, esse sujeito apresentava inventário fonético completo. O sistema fonológico estava composto por plosivas, fricativas, africadas, nasais e líquidas laterais. A líquida nãolateral /r/ em Onset $(11,11 \%)$ e em Coda (0\%) não estavam adquiridas. Em Onset era substituído por [1] (77,78\%), e apagado em 11,11\%; enquanto que, em Coda, era omitido em $74,07 \%$ das vezes, substituído por [y] (14,82\%) e [1] (11,11\%). Havia redução de todos os Onsets Complexos (OC).

A líquida não-lateral /r/ foi escolhida como alvo de tratamento por ser o único som não adquirido no sistema fonológico da criança. No tratamento com o /r/ em OM, foram selecionadas as seguintes palavras-alvo: [ba'rata], [kora'sãw], [se'nora], [ku'ruza], [mo'rãgu], [t fi'zora].

$\mathrm{Na}$ análise do ambiente favorável para este caso tratado, foi verificado que: considerando-se a líquida não-lateral /r/ nas palavras selecionadas, todas se encontravam na posição de OM, a posição mais favorável; quanto à tonicidade, apenas nas palavras-alvo [ba'rata], [ku'ruza] e [mo'rãgu] o /r/ encontrava-se em ambiente favorável (sílaba tônica); e, quanto ao contexto antecedente e seguinte, nenhuma das palavras encontrava-se em ambiente favorável.

Quadro 1. Modelo ABAB - Retirada e Provas Múltiplas

\begin{tabular}{|c|c|c|}
\hline $\begin{array}{l}\text { COLETA DOS } \\
\text { DADOS DE } \\
\text { FALA (A1) }\end{array}$ & $\begin{array}{l}\text { Sessão } 1 \\
\text { Sessão } 2 \\
\text { Sessão } 3\end{array}$ & $\begin{array}{l}\rightarrow \text { PROVA DE GENERALIZAÇÃO (AFC) } \\
\rightarrow \text { PROVA DE GENERALIZAÇÃO (AFC) } \\
\text { Amostra da fala espontânea }\end{array}$ \\
\hline \multicolumn{3}{|l|}{$\downarrow$} \\
\hline \multirow{5}{*}{$\begin{array}{l}1^{\circ} \mathrm{CICLO} \text { DE } \\
\text { TRATAMENTO }\end{array}$} & Sessão 1 & $\begin{array}{l}\text { - PROVA ALVO BÁSICA } 1 \text { (gravada) } \\
\text { * imitação retardada * }\end{array}$ \\
\hline & $\begin{array}{l}\text { Sessão } 2 \\
\text { Sessão } 3 \\
\text { Sessão } 4\end{array}$ & $\begin{array}{l}\text { * Sessão Terapêutica } 1 \\
\text { * Sessão Terapêutica } 2 \\
\text { * Sessão Terapêutica } 3\end{array}$ \\
\hline & Sessão 5 & $\begin{array}{l}\rightarrow \text { PROVA ALVO BÁSICA } 2 \text { (gravada) } \\
\text { * menor imitação * }\end{array}$ \\
\hline & $\begin{array}{l}\text { Sessão } 6 \\
\text { Sessão } 7 \\
\text { Sessão } 8\end{array}$ & $\begin{array}{l}\text { * Sessão Terapêutica } 4 \\
\text { * Sessão Terapêutica } 5 \\
\text { * Sessão Terapêutica } 6\end{array}$ \\
\hline & Sessão 9 & $\begin{array}{l}\rightarrow \text { PROVA ALVO BÁSICA } 3 \text { (gravada) } \\
\text { * sem imitação * }\end{array}$ \\
\hline \multicolumn{3}{|l|}{$\downarrow$} \\
\hline $\begin{array}{l}\text { PERÍODO } \\
\text { DE RETIRADA } \\
\text { (A2) }\end{array}$ & $\begin{array}{l}\text { Sessão } 1 \\
\text { Sessão } 2 \\
\text { Sessão } 3 \\
\text { Sessão } 4 \\
\text { Sessão } 5\end{array}$ & $\begin{array}{l}\text { * PROVA DE GENERALIZAÇÃO - } 1 \text { (AFC) } \\
\text { * PROVA DE GENERALIZAÇÃO - } 1 \text { (AFC) } \\
\text { * Amostra da fala espontânea } \\
\text { * PROVA DE GENERALIZAÇÃO - } 2 \text { (AFC) } \\
\text { * PROVA DE GENERALIZAÇÃO - } 2 \text { (AFC) }\end{array}$ \\
\hline
\end{tabular}


Quadro 2. Comparação do sistema fonológico inicial e final, com indicação do número de sons adquiridos após o tratamento

\begin{tabular}{|c|c|c|c|c|c|c|c|c|}
\hline \multirow[t]{2}{*}{ Posição } & \multicolumn{3}{|c|}{ Sistema Fonológico Inicial } & \multirow{2}{*}{$\begin{array}{c}\text { Som } \\
\text { estimulado }\end{array}$} & \multicolumn{3}{|c|}{ Sistema Fonológico Final } & \multirow{2}{*}{$\begin{array}{l}\mathrm{N} \text { de sons } \\
\text { adquiridos }\end{array}$} \\
\hline & SA & SPA & SNA & & SA & SPA & SNA & \\
\hline Ol & $\begin{array}{l}/ \mathrm{p} /, / \mathrm{b} /, / \mathrm{t} /, / \mathrm{d} /, / \mathrm{k} /, / \mathrm{g} /, / \mathrm{f} /, / \mathrm{v} /, / \mathrm{s} / \text {, } \\
/ \mathrm{z} /, / \mathrm{s} /, / 3 /, / \mathrm{m} /, / \mathrm{n} /, / \mathrm{h} /, / / \mathrm{l} \\
/ \mathrm{N} /, / \mathrm{R} /,[\mathrm{t}] \text { e e [dz] }\end{array}$ & & & /r/ em OM & $\begin{array}{l}/ \mathrm{p} /, / \mathrm{b} /, / \mathrm{t} /, / \mathrm{d} /, / \mathrm{k} /, / \mathrm{g} /, / \mathrm{f} /, / \mathrm{v} /, / \mathrm{s} /, \\
/ \mathrm{z} /, / \mathrm{s} /, / 3 /, / \mathrm{m} /, / \mathrm{n} /, / \mathrm{h} /, / / / \\
/ \mathrm{N} /, / \mathrm{R} /,[\mathrm{t}] \text { e e [dz] }\end{array}$ & & & 0 \\
\hline OM & $\begin{array}{l}/ \mathrm{p} /, / \mathrm{b} /, / \mathrm{t} /, / \mathrm{d} /, / \mathrm{k} /, / \mathrm{g} /, / \mathrm{ff} /, / \mathrm{v} /, \\
/ \mathrm{s} /, / \mathrm{z} /, / \mathrm{s} /, / 3 /, / \mathrm{m} /, / \mathrm{n} /, / \mathrm{g} /, \\
/ \mathrm{l} /, / \mathrm{N} /, / \mathrm{R} /,[\mathrm{t}] \text { ] e [dz] }\end{array}$ & & $/ r /$ & & $\begin{array}{l}\mathrm{p} /, / \mathrm{b} /, / \mathrm{t} /, / \mathrm{d} /, / \mathrm{k} /, / \mathrm{g} /, / \mathrm{ff} /, / \mathrm{v} /, / \mathrm{s} / \\
/ \mathrm{z} /, / \mathrm{s} /, / 3 /, / \mathrm{m} /, / \mathrm{n} /, / \mathrm{l} /, / / \mathrm{l} \\
/ \mathrm{N} /, / \mathrm{R} /, / \mathrm{r} /,[\mathrm{t}] \text { e e [dz] }\end{array}$ & & & 1 \\
\hline $\mathrm{CM}$ & $/ \mathrm{s} /$ & & $/ r /$ & & /s/ & $/ \mathrm{r} /$ & & 0 \\
\hline $\mathrm{CF}$ & $/ \mathrm{s} /$ & & $|r|$ & & $/ \mathrm{s} / \mathrm{e} / \mathrm{r} /$ & & & 1 \\
\hline
\end{tabular}

Legenda: SA: Sons Adquiridos; SPA: Sons Parcialmente Adquiridos; SNA: Sons Não Adquiridos; OI: Onset Inicial; OM: Onset Medial; CM: Coda Medial; CF: Coda Final

Tabela 1. Ocorrências de produções corretas em ambientes favoráveis e não-favoráveis quanto à tonicidade nas PABs no primeiro ciclo de tratamento

\begin{tabular}{|c|c|c|c|c|c|c|c|c|c|c|c|c|c|c|}
\hline \multirow[b]{2}{*}{$\mathrm{N}$} & \multicolumn{3}{|c|}{ PAB 1} & \multirow[b]{2}{*}{$\%$} & \multicolumn{5}{|c|}{ PAB 2} & \multicolumn{4}{|c|}{ PAB 3} & \multirow[b]{2}{*}{$\%$} \\
\hline & Fav & $\%$ & Não-Fav & & $n$ & Fav & $\%$ & Não-Fav & $\%$ & $\mathrm{n}$ & Fav & $\%$ & Não-Fav & \\
\hline 23 & 12 & 52,17 & 4 & 17,39 & 21 & 12 & 57,14 & 5 & 23,81 & 22 & 9 & 40,91 & 9 & 40,91 \\
\hline
\end{tabular}

Legenda: PAB - Prova Alvo Básica; $\mathrm{n}$ - número de produções; Fav - ambiente favorável; Não-Fav - não favorável

A Tabela 1 mostra as ocorrências de produções corretas em ambientes favoráveis e não-favoráveis quanto à tonicidade nas PABs no primeiro ciclo de tratamento.

Durante o ciclo de tratamento, na análise das PABs, quanto às aquisições com relação à tonicidade, observou-se que os ambientes mais favoráveis para aquisição estavam nas palavras-alvo - [ba'rata], [ku'ruza] e [mo'rãgu] - sendo que [kora'sãw], [se'nora] e [t $\int i$ 'zora] eram menos favoráveis para a produção da líquida. Dentre essas, a palavra-alvo [tSði'zora] foi realizada incorretamente em todas as PABs. De 23 possibilidades de ocorrência na PAB 1 houve $12(57,17 \%)$ produções em AF e $4(17,39 \%)$ em não favoráveis. A palavra [ba'rata] ocorreu 5 vezes, [ku'ruza] 4, e [mórãgu] 3. Na PAB 2, de 21 possibilidades, $12(57,14 \%)$ produções estavam em $\mathrm{AF}$ e $5(23,81 \%)$ em ambientes não favoráveis, sendo que as palavras que mais ocorreram foram [mórãgu] (5 vezes), [ba'rata] (4) e [ku'ruza] (3). Com relação a PAB 3, houve 22 possibilidades, $9(40,91 \%)$ estavam em AF e 9 $(40,91 \%)$ em ambientes não favoráveis (tabela 1$)$. A palavra [ba'rata] ocorreu 5 vezes, [ku'́ruza] e [mórãgu] 2 vezes.

A Tabela 2 apresenta as ocorrências de produções corretas em ambientes não-favoráveis quanto ao contexto antecedente e seguinte nas PABs no primeiro ciclo de tratamento.

$\mathrm{Na}$ análise das PABs verificou-se que, quanto ao contexto antecedente e seguinte, nenhuma palavra trabalhada era favorável, sendo que houve $18(81,82 \%)$ produções corretas, como apresentado na tabela 2. Na PAB1, a palavra [bárata] ocorreu 5 vezes, [ku'ruza] 4, e [mórãgu] 3, na PAB 2 a palavra [mo'rãgu] apresentou 5 ocorrências, [ba'rata] 4, [ku'ruza] e [sénora] 3. Por fim, na PAB 3 as palavras mais realizadas foram: [kora'sãw] 6 vezes, [ba'rata] 5 e [se'nora] 3.

Com a reavaliação fonológica, observou-se que, no sistema fonológico geral, o sujeito adquiriu a líquida não-lateral /r/ em Onset (85\%), e em Coda, ficou parcialmente adquirida (62\%). Em Onset, era substituído por [1] (12,50\%), e
Tabela 2. Ocorrências de produções corretas em ambientes nãofavoráveis quanto ao contexto antecedente e seguinte nas PABs no primeiro ciclo de tratamento.

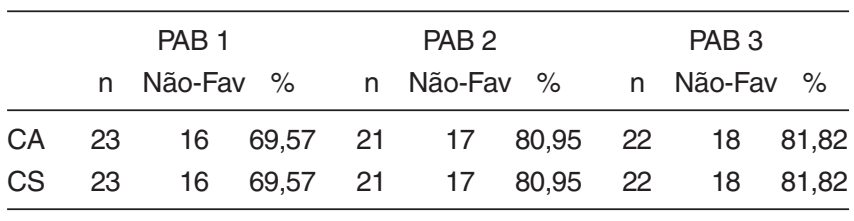

Legenda: PAB - Prova Alvo Básica; $\mathrm{n}$ - número de produções; Não-Fav - não favorável; CA - contexto antecedente; CS -contexto seguinte

omitido em 2,50\%; enquanto que, em Coda, era omitido em $38 \%$ das vezes. Quanto ao OC, alguns foram adquiridos, como por exemplo, /pl/ e /fr/, que são outras variáveis e são mais complexas.

\section{DISCUSSÃO}

Serão comentados os AF do sujeito com DF, correlacionando os achados descritos na literatura quanto aos AF na aquisição normal.

No sistema fonológico inicial deste sujeito, estava ausente apenas a líquida não-lateral /r/, como é descrito na literatura $^{(1-2)}$ esse fonema é o último a se estabilizar na fala da criança. Pôde-se observar que no sistema fonológico final, o /r/ estava adquirido nas posições de $\mathrm{OM}$ e $\mathrm{CF}$ e parcialmente adquirido em CM, estando de acordo com a literatura compulsada $^{(2)}$ que refere ser esta posição a última a ser adquirida.

Verificou-se, quanto à tonicidade, que a maior parte das produções corretas apresentavam-se em AF, como pôde ser observado nas palavras-alvo: [ba'rata], [ku'ruza] e [mo'rãgu], em que o /r/ apresentava-se em sílaba tônica. Esses resultados concordam com os achados de um estudo ${ }^{(8)}$ que apontou a sílaba tônica como mais facilitadora para a produção do 
som, enquanto que a posição postônica seria menos facilitadora. Outro estudo ${ }^{(9)}$ verificou que a sílaba postônica favoreceu a estabilização do /r/ em sua aquisição. Outra autora $^{(11)}$ encontrou as sílabas tônica e pretônica como fatores neutros para aquisição da líquida não-lateral.

Com relação ao contexto antecedente, o sujeito não apresentou nenhuma palavra-alvo em AF. Alguns estudos ${ }^{(8,11)}$ consideram a vogal /i/ como mais facilitadora para aquisição do fonema $/ r /$. Os contextos antecedentes encontrados nas palavras-alvo selecionadas para o sujeito eram: /a/, /o/ e /u/, sendo /a/ e /o/ as que apresentaram maior incidência de produções corretas. Alguns autores ${ }^{(10-11)}$, consideram as vogais /a/ e /o/ como fatores neutros para aquisição; outros ${ }^{(9)}$ afirmam que nenhuma das vogais favoreceu a produção do som-alvo /r/. Apesar dos autores não considerarem /a/ e /o/ como facilitadores, tais ambientes foram favoráveis, principalmente com a vogal /o/, nas palavras [kora'sãw] e [mórãgu], que apresentaram maior número de realizações corretas.

Quanto ao contexto seguinte, as palavras utilizadas foram desfavoráveis para aquisição. Alguns autores ${ }^{(8,11)}$ verificaram que a vogal /i/ facilitava a aquisição do fonema /r/. Os contextos seguintes encontrados nas palavras-alvo selecionadas eram: /a/ e /u/, sendo /a/ a vogal de maior incidência de realizações corretas. Entre as pesquisas realizadas, uma aponta ${ }^{(11)}$ essa vogal como fator neutro, outra ${ }^{(9)}$, como desfavorável. Os achados das pesquisas não consideram /a/ como facilitadora, mas esse ambiente foi relevante para a aquisição do rótico. As palavras utilizadas em terapia foram [kora'sãw], [ba'rata], [se'nora], [t fi'zora] e [mo'rãgu], sendo as maiores ocorrências [kora'sãw], [ba'rata] e [mórãgu].

As palavras usadas para tratar o sujeito foram: [ba'rata], [kora'sãw], [se'nora], [ku'ruza], [mo'rãgu] e [tfi'zora], sendo que as palavras [ba'rata], [kora'sãw], [ku'ruza] e [mo'rãgu] encontram-se no padrão $5^{(10)}$ e [se'nora] e [t $\int i^{\prime}$ zora] no padrão 6. Dentre as palavras do padrão 6 não existe maior facilitação entre elas. Todavia, no padrão 5 a palavra [ku'ruza] é mais favorável que [ba'rata] e [kora'sãw]. Os padrões descritos acima pertencem a um estudo ${ }^{(10)}$ no qual foram sugeridas palavras-estímulo selecionadas, combinadas e reunidas em 12 padrões de facilitação para o vocábulo /r/. Essa autora sugere, ainda, que no padrão 1, mais facilitador, estão

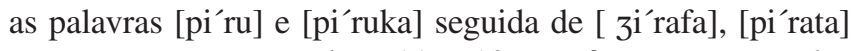
entre outras. E no padrão 11 e 12 não foram encontradas palavras na língua portuguesa. Embora o sujeito não tenha sido tratado com palavras dos primeiros padrões estudados, essas deveriam ser selecionadas quando possível, mas, mesmo assim, as palavras-alvo escolhidas para o tratamento foram eficazes para a aquisição da líquida não-lateral /r/. Porém, pode-se pensar que, se as palavras selecionadas fossem dos padrões 1 ou 2, a aquisição do som-alvo seria mais rápida.

Atualmente, não se tem conhecimento sobre pesquisas a respeito dos ambientes favoráveis em sujeitos com desvio fonológico falantes do Português. O modelo ABAB-Retirada e Provas Múltiplas, utilizado nesse estudo, pode ter favorecido a análise dos ambientes favoráveis, pois no tratamento utiliza-se apenas um som-alvo em cada ciclo. Seria importante que outras pesquisas analisassem os ambientes favoráveis no tratamento para outros modelos, como Oposições Máximas, ou Modelo de Ciclos.

\section{COMENTÁRIOS FINAIS}

Este trabalho teve como objetivo analisar a influência das variáveis lingüísticas (ambiente favorável) no tratamento de um sujeito com desvio fonológico evolutivo, e permitiu as seguintes conclusões:

- após o tratamento, adquiriu a líquida não-lateral /r/ em $\mathrm{OM}$, generalizando o fonema a outras palavras, além dos alvos de tratamento; em Coda, o rótico /r/ ficou parcialmente adquirido; e quanto ao OC, realizou /pl/ e /fr/;

- considerando os ambientes favoráveis, quanto à tonicidade, houve um maior número de produções corretas de /r/, quando este se encontrava em sílaba tônica; com relação aos contextos antecedente e seguinte, não houve palavras em AF, porém, as vogais /a/ (contexto seguinte) e /o/ (contexto antecedente) mostraram-se favoráveis para a aquisição do rótico;

- embora não tenham sido selecionadas palavras-alvo nos ambientes mais favoráveis, as palavras selecionadas, apesar de estarem nos padrões 5 e 6 , foram efetivas para a aquisição do som-alvo tratado em um ciclo de tratamento.

\begin{abstract}
Favorable environments are contexts that make the acquisition of the segment treated easier. They can be analyzed under the following aspects: tonicity, preceding context and following vowel. This case report had the aim to analyze the influence of linguistic variables (favorable environment) at the treatment of a patient suffering from phonological disorder. A male patient, aged 6:8 at the beginning of the treatment, treated by the Model ABAB-Withdrawal and Multiple Probes participated in this study. Pre and post-treatment speech data and the Basic Target Proof of the first treatment cycle were analyzed. The acquisitions regarding the treatment target the non-lateral liquid $/ \mathrm{r} /$ - and the favorable environments to the acquisition regarding tonicity and contexts (preceding and following) were analyzed in the target words selected for the therapy. It was observed that the $/ \mathrm{r} /$, in the select target words, was not found in more favorable environments; in general, it was found in neutral or less favorable environments. However, generalization was verified concerning the non-lateral liquid /r/ acquisition in medial onset, and the phoneme production in the select target words was effective, with emphasis to the production of the words [ba'rata], [kóruza] and [mórãgu], which had a larger number of occurrences.
\end{abstract}

KEYWORDS: Speech; Speech disorders/therapy; Speech therapy; Child; Articulation disorders 


\section{REFERÊNCIAS}

1. Lamprecht RR. A aquisição da fonologia do português na faixa etária dos 2:9 aos 5:5. Porto Alegre. Letras de Hoje. 1993;28(2):99-106.

2. Miranda ARM. A aquisição das líquidas não-laterais no português do Brasil. Letras de Hoje. 1998;33(2):123-31.

3. Keske-Soares M. Terapia fonoaudiológica fundamentada na hierarquia implicacional dos traços distintivos aplicada em crianças com desvios fonológicos [tese]. Porto Alegre: Faculdade de Letras da Pontifícia Universidade Católica do Rio Grande do Sul; 2001.

4. Wertzner HF. Fonologia: desenvolvimento e alterações. In: Ferreira LP, Befi-Lopes DM, Limongi SCO, organizadores. Tratado de fonoaudiologia. São Paulo: Roca; 2004.

5. Vidor DCGM. A aquisição das líquidas não-laterais por crianças com desvios fonológicos evolutivos: descrição, análise e comparação com desenvolvimento normal [tese]. Porto Alegre: Faculdade de Letras da Pontifícia Universidade Católica do Rio Grande do Sul; 2000.

6. Mota HB. Os padrões de erros nas alterações de fala analisados pelo modelo implicacional de complexidade de traços. Pró-Fono. 1998;10(1):73-80.

7. Donicht G. A generalização obtida a partir do tratamento com os róticos em dois modelos de terapia fonoaudiológica para crianças com desvio fonológico [monografia]. Santa Maria: Universidade Federal de Santa Maria; 2004.
8. Mezzomo CL, Ribas LP. Sobre a aquisição das líquidas. In: Lamprecht RR. Aquisição fonológica do português: perfil de desenvolvimento e subsídios para terapia. São Paulo: Artmed; 2004.

9. Hernandorena CLM, Lamprecht RR. A aquisição das consoantes líquidas do português. Letras de Hoje. 1997;32(4):7-22.

10. Gonçalves CS. Variáveis lingüísticas facilitadoras na reabilitação fonológica das líquidas não-laterais [tese]. Porto Alegre: Faculdade de Letras da Pontifícia Universidade Católica do Rio Grande do Sul; 2002.

11. Miranda ARM. A aquisição do "r": uma contribuição à discussão sobre seu status fonológico [tese]. Porto Alegre: Faculdade de Letras da Pontifícia Universidade Católica do Rio Grande do Sul; 1996.

12. Yavas M, Hernandorena CLM, Lamprecht RR. Avaliação fonológica da criança: reeducação e terapia. Porto Alegre: Artes Médicas; 1992.

13. Rodrigues EJB. Discriminação aditiva: normas para avaliação de crianças de 5 a 9 anos. São Paulo: Cortez; 1981.

14. Bernhardt B. Development implications of nonlinear phonological theory. Clin Linguist Phon. 1992;6(4):259-81.

15. Tyler AA, Figursky GR. Phonetic inventory changes after treating distinctions along an implicational hierarchy. Clin Linguist Phon. 1994;8(2):91-108. 